\title{
Amniotic fluid-derived multipotent stromal cells drive diabetic wound healing through modulation of macrophages
}

\author{
Bibi S. Subhan ${ }^{\dagger}$, Jennifer Kwong ${ }^{\dagger}$, Joseph F. Kuhn, Arie Monas, Sonali Sharma and Piul S. Rabbani* (D)
}

\begin{abstract}
Background: Cutaneous wounds in patients with diabetes exhibit impaired healing due to physiological impediments and conventional care options are severely limited. Multipotent stromal cells (MSCs) have been touted as a powerful new therapy for diabetic tissue repair owing to their trophic activity and low immunogenicity. However, variations in sources and access are limiting factors for broader adaptation and study of MSC-based therapies. Amniotic fluid presents a relatively unexplored source of MSCs and one with wide availability. Here, we investigate the potential of amniotic fluid-derived multipotent stromal cells (AFMSCs) to restore molecular integrity to diabetic wounds, amend pathology and promote wound healing.

Method: We obtained third trimester amniotic fluid from term cesarean delivery and isolated and expanded MSCs in vitro. We then generated $10 \mathrm{~mm}$ wounds in Lepr ${ }^{\mathrm{db} / \mathrm{db}}$ diabetic mouse skin, and splinted them open to allow for humanized wound modeling. Immediately after wounding, we applied AFMSCs topically to the sites of injuries on diabetic mice, while media application only, defined as vehicle, served as controls. Post-treatment, we compared healing time and molecular and cellular events of AFMSC-treated, vehicle-treated, untreated diabetic, and non-diabetic wounds. A priori statistical analyses measures determined significance of the data.
\end{abstract}

Result: Average time to wound closure was approximately 19 days in AFMSC-treated diabetic wounds. This was significantly lower than the vehicle-treated diabetic wounds, which required on average 27.5 days to heal $(p<0.01)$, and most similar to time of closure in wild type untreated wounds (an average of around 18 days). In addition, AFMSC treatment induced changes in the profiles of macrophage polarizing cytokines, resulting in a change in macrophage composition in the diabetic wound bed. We found no evidence of AFMSC engraftment or biotherapy induced immune response.

Conclusion: Treatment of diabetic wounds using amniotic fluid-derived MSCs encourages cutaneous tissue repair through affecting inflammatory cell behavior in the wound site. Since vehicle-treated diabetic wounds did not demonstrate accelerated healing, we determined that AFMSCs were therapeutic through their paracrine activities. Future studies should be aimed towards validating our observations through further examination of the paracrine potential of AFMSCs. In addition, investigations concerning safety and efficacy of this therapy in clinical trials should be pursued.

Keywords: Amniotic fluid multipotent stromal cells, Diabetic wounds, Cellular therapy

${ }^{*}$ Correspondence: piul.rabbani@nyulangone.org

†Bibi S. Subhan and Jennifer Kwong contributed equally

Hansjörg Wyss Department of Plastic Surgery, New York University School

of Medicine, 540 First Avenue, New York 10016, USA

(c) The Author(s) 2021. This article is licensed under a Creative Commons Attribution 4.0 International License, which permits use, sharing, adaptation, distribution and reproduction in any medium or format, as long as you give appropriate credit to the original author(s) and the source, provide a link to the Creative Commons licence, and indicate if changes were made. The images or other third party material in this article are included in the article's Creative Commons licence, unless indicated otherwise in a credit line to the material. If material is not included in the article's Creative Commons licence and your intended use is not permitted by statutory regulation or exceeds the permitted use, you will need to obtain permission directly from the copyright holder. To view a copy of this licence, visit http://creativeco mmons.org/licenses/by/4.0/. The Creative Commons Public Domain Dedication waiver (http://creativecommons.org/publicdomain/ zero/1.0/) applies to the data made available in this article, unless otherwise stated in a credit line to the data. 


\section{Background}

The urgent need for effective treatment of chronic diabetic ulcers has seen a surge in utilization of mesenchymal cells with primitive stem-like properties. Diabetes and associated complications affect $9.4 \%$ of the population of the United States, or 1 in every 11 individuals [1]. Chronic non-healing ulcers are one of the most conspicuous manifestations of this disease, costing an estimated $\$ 1.38$ billion annually [2]. This monetary value, however, does not account for psychosocial and economic impacts on the quality of life for both patients and caregivers. Currently, most available healthcare options address wound management, but not therapy of these diabetic wounds. The search for innovative and effective solutions that can target the complex pathology of diabetic ulcers has recently uncovered the potential of mesenchymal progenitor cells.

Several studies have explored the capability of mesenchymal progenitor cells derived from adipose tissue, bone marrow, umbilical cord among other sources for tissue repair and regeneration through treatment of cardiac [3], hepatic [4], renal [5], ischemic injuries [6, 7] and cutaneous wounds [8] to name a few. In our previous work, we have demonstrated the use of bone marrowderived multipotent stromal cells for effectively promoting closure of mouse type II diabetic wounds [9]. Among the potential cell types being considered for therapy, an under-utilized source is human amniotic fluid-derived multipotent stromal cells (AFMSCs). Amniotic fluid, composed mainly of water, also contains a fluctuating solute content of essential nutrients and immune effectors, and a population of cells that upon adherent culture enrich for mesenchymal progenitors [10]. This fluid is clinically utilized for pre-natal analysis and diagnostics, then typically discarded at childbirth, but is in fact an abundant and widely available source of mesenchymal progenitor cells with remedial potential.

A liter of amniotic fluid contains approximately 7 million mononucleated cells [11], while $\sim 5 \times 10^{5}$ cells per liter demonstrate plastic adherence in cell culture conditions [12]. These amniotic fluid cells have easy expansion in readily available standard reagents [10,13], and in just 4 weeks of growth, 180 million cells with AFMSC morphology can be derived from $2 \mathrm{~mL}$ of amniotic fluid [14]. Adverse events, such as tumor formation, due to administration of AFMSCs have not been reported according to the literature, an indication of the safety of their allogeneic use $[15,16]$. Additionally, there are no ethical stipulations as for embryonic stem cells, since they are obtained from an adult source [17]. Despite their source, in vitro analysis of AFMSCs have characterized them with markers of both adult and embryonic stem cells $[18,19]$. Although inherent pluripotent potential of these
AFMSCs has not been unanimously established, compared to adult stem cells they do have greater proliferation and more widespread differentiation [10, 20-22].

AFMSCs are a heterogeneous population but demonstrate capacities similar to adult mesenchymal progenitor cells of reliable differentiation along mesenchymal lineages like osteogenic, adipogenic and chrondrogenic [19, $21,23]$. These cells have high proliferative capacity and demonstrate multipotency, much like adult mesenchymal multipotent cells. The cells do not require feeder layers and reliably proliferate in mesenchymal type cell culture media. Full-term and early-term AFMSCs show similar characteristics, pointing to full term amniotic fluid as an easily accessible source of therapeutic cells [13, 24].

A few studies have reported efficacy of AFMSCs in wound healing models via modulation of the inflammatory phase of healing $[25,26]$. The majority of studies using AFMSCs have investigated cardiovascular, gastrointestinal, neural, respiratory and muskulo-skeletal tissue repair models [20]. Their potential for fulfilling the public health need of an effective therapy for accelerating treatment of non-healing diabetic wounds requires careful investigation. Previous molecular analyses of diabetic wounds have related deficits in key physiological elements and events including growth factor and macrophage responses, angiogenic activation, and overall cellular and extracellular matrix migration and remodeling [27]. These impairments result in an overall chronically inflamed, non-healing phenotype of the diabetic wounds. Therapy aimed at diabetic wounds should have the ability to coordinate molecular events towards normal wound healing stages.

In this study, we cultured heterogeneous mesenchymal progenitor cells from human full-term gestation amniotic fluid, and assessed the efficacy of these AFMSCs in promoting wound closure in a type II diabetic full-thickness pre-clinical wound model. We find that a single topical application of AFMSCs is sufficient to drive tissue repair, and to reduce pathologic healing time. In particular, we identified polarization of wound site macrophages towards anti-inflammatory phenotypes in response to AFMSC administration, to accelerate diabetic wound closure.

\section{Methods \\ AFMSC culture}

Collection of amniotic fluid and derived products was approved by the Institutional Review Board at New York University School of Medicine. Amniotic fluid was collected at the time of scheduled, term cesarean delivery with the patient's informed consent (study\# i15-01269, New York, NY, USA) and cultured per published protocols to enrich for AFMSCs [13]. Briefly, amniotic fluid 
stem cells were isolated from the fluid and cultured in monolayer in medium consisting of $20 \%$ Chang medium D (Irvine Scientific, Irvine, CA, USA), MEM-alpha GlutaMAX (Life Technologies, Carlsbad, CA, USA), 15\% embryonic stem cell-qualified fetal bovine serum (Life Technologies, Carlsbad, CA, USA) and $100 \mu \mathrm{g} / \mathrm{mL}$ Normocin (InvivoGen, San Diego, CA, USA), then cryopreserved at passage 2 . Following thawing, cells were incubated at $37{ }^{\circ} \mathrm{C}, 5 \% \mathrm{CO}_{2}, 95 \%$ humidity until $80 \%$ confluent. We detached cells from the plate using Accutase (Thermo Scientific, Waltham, MA, USA) for further passages or experiments. For the design of this study, AFMSCs from 3 separate batches of amniotic fluid were combined in cell culture.

\section{Animal protocol}

All animal protocols were approved by the New York University School of Medicine Institutional Animal Care and Use Committee. We obtained male and female diabetic $\left(\mathrm{Lepr}^{\mathrm{db} / \mathrm{db}}\right)$ mice, aged 6-8 weeks, from The Jackson Laboratory (Bar Harbor, ME, USA). All Lepr ${ }^{\mathrm{db} / \mathrm{db}}$ mice used in the experiments had blood glucose concentrations of $>400 \mathrm{mg} / \mathrm{dL}$. We obtained C57BL/6N wild type (WT) mice from Taconic Biosciences (Rensselaer, NY, USA). We housed these mice in a temperaturecontrolled, virus-free barrier animal facility with a 12-h light/12-h dark cycle and maintained them on chow diet and water ad libitum.

\section{Preclinical wounding model}

Following sedation with $2 \%$ isoflurane, we used the foot pad pinch test to confirm that each mouse was properly anesthetized. We used an established murine model of excisional wound healing [28]. Briefly, we first removed hair on the mouse dorsum using a hair trimmer and Nair (Church \& Dwight, Princeton, NJ, USA). We created paired 10-mm full-thickness wounds extending through the panniculus carnosus on the dorsum of the mice using a punch biopsy tool, and then splinted the wounds open with $0.6 \mathrm{~mm}$ thick silicone stents of $10 \mathrm{~mm}$ inner diameter and $20 \mathrm{~mm}$ outer diameter (silicone sheets from Grace Bio-Labs, Bend, OR, USA). Once positioned over the wound, we secured the stent to the full thickness of the dorsal skin with interrupted 4-0 braided sutures (Henry Schein, Inc., Melville, NY, USA). We placed the sutures to ensure that they do not disrupt the boundary of the wound. We then covered the stented wounds with occlusive dressing with a $10 \mathrm{~mm}$ window to allow air exchange, while preventing changes in wound dimensions due to gnawing or pulling from the mice. Analgesics provided for 3 consecutive days post-operation aid in preventing mice from disrupting the wound healing. We used standardized photographs at regular intervals post-op to photometrically analyze the percent wound remaining, calibrating against the internal diameter of the $10 \mathrm{~mm}$ silicone stent to correct for magnification, perspective, or parallax effects. We recorded time to wound closure as the number of days for complete scab detachment and resemblance to intact skin by gross visual inspection. We calculated percent wound remaining [(unhealed wound area)/(original wound area)] using digital measurements of wound photographs (Adobe Systems, San Jose, CA, USA). We used area under the curve (GraphPad software) to assess the wound burden [29]. Wound photographing, calculation of unhealed wound areas and wound burden using photographs were all blinded.

\section{AFMSC administration}

At passage 5, we detached cells using Accutase, then centrifuged at $300 \times g$ and resuspended in AFMSC media at $5 \times 10^{4}$ cells $/ \mu \mathrm{L}$. Using a sterile micropipette, we administered $10 \mu \mathrm{L}$ of AFMSC suspension $\left(5 \times 10^{5}\right.$ cells total $)$ directly into the wound, immediately after excision and stenting. For cell tracing studies, we incubated the cells with $1,1^{\prime}$-Dioctadecyl-3,3,3',3'Tetramethylindocarbocyanine Perchlorate (DiI, D3911, Invitrogen) at a final concentration of $4 \mathrm{ng} / \mu \mathrm{L}$. Following a $2 \mathrm{~h}$ incubation, we washed cells 3 times with fresh, warmed $1 \times$ PBS prior to detachment. Before detaching cells, we confirmed and photographed DiI labeling using a Cytation 5 (Biotek, VT, USA). The treatment administration was blinded, as well as subsequent monitoring and wound analysis to prevent any subjective biases.

\section{In vivo AFMSC imaging}

Prior to AFMSC administration, we positioned anesthetized mice in an In Vivo Imaging System (IVIS) Lumina III (Perkin Elmer, MA, USA) and captured fluorescence at excitation $560 \mathrm{~nm}$ and emission $610 \mathrm{~nm}$. We re-imaged the wounds on the mice immediately following administration of DiI-labeled AFMSCs. We continued imaging at $6,12,24$ and $48 \mathrm{~h}$ post-administration and analyzed the data using Living Image software (Perkin Elmer, MA, USA). Mice were identified with numbers and treatments were blinded.

\section{Wound tissue harvest}

For histological analyses, we excised the wound with a $5 \mathrm{~mm}$ margin diameter, dissected along the cranialcaudal axis and fixed the skin wound tissue in $4 \%$ paraformaldehyde overnight at $4{ }^{\circ} \mathrm{C}$. For wounds treated with DiI-labeled AFMSCs, following overnight fixation to preserve any fluorescent signal, we cryopreserved the tissue using $15 \%$ and $30 \%$ sucrose sequentially, then embedded in optimal cutting temperature medium (Neg 
50, FisherScientific, CA, USA). For those treated with non-labeled AFMSCs, wound tissue samples underwent routine histologic processing for embedding in paraffin and sectioning at $5 \mu \mathrm{m}$ thickness. We used deparaffinized skin-tissue sections for hematoxylin and eosin staining, performed by the Experimental Pathology Laboratory at NYU School of Medicine.

\section{Dil-label detection in wound tissue sections}

We dried slides with $20 \mu \mathrm{m}$ frozen wound tissue sections for $60 \mathrm{~min}$ at room temperature, prior to rehydration in $1 \times$ PBS and 2 further washes. We counterstained the sections using Hoechst 33342 and mounted with Prolong Gold Antifade Mountant (ThermoFisher Scientific, CA, USA). We analyzed and photographed sections on a Nikon Ti2E (Nikon, NY, USA).

\section{Immunostaining}

After deparaffinization of wound tissue sections, we performed heat-mediated antigen retrieval in a $\mathrm{pH} 6$ sodium citrate buffer (Dako). Then we blocked and permeabilized using a 5\% normal donkey serum (017-000-121, Jackson ImmunoResearch) and 0.01\% Triton-X (9002-93-1, Sigma Aldrich) in PBS $/ 0.2 \%$ bovine serum albumin $/ 0.75 \%$ glycine. We immunostained with primary antibodies for rabbit anti-mouse Arginase 1 (16,001-1-AP, Proteintech) and rat anti-mouse F4/80 (ab6640, Abcam), and donkey anti-rabbit and donkey anti-rat IgG secondary antibodies, conjugated to Alexafluor 594 and Alexafluor 488 , respectively. We counterstained the sections using Hoechst 33342 and mounted with Prolong Gold Antifade Mountant (ThermoFisher Scientific, CA, USA). We analyzed and photographed sections on a Nikon Ti2E (Nikon, NY, USA).

\section{RNA isolation and quantitative PCR}

We lysed AFMSCs on the culture plates using Trizol, per the manufacturer's guidelines (ThermoFisher Scientific, CA, USA). For wound tissues, we homogenized the tissue in Trizol as well, using ceramic beads in a Beadmill for 2 cycles of $30 \mathrm{~s} 6 \mathrm{~m} / \mathrm{s}$ runs (ThermoFisher Scientific, CA, USA). We added $200 \mu \mathrm{L}$ chloroform per $1 \mathrm{~mL}$ Trizol, agitated the samples and allowed phase separation for $5 \mathrm{~min}$ at room temperature. Following centrifugation at $12,000 \times g$ for $15 \mathrm{~min}$ at $4{ }^{\circ} \mathrm{C}$, we removed the aqueous phase containing RNA and precipitated using isopropanol. We loaded the precipitated RNA onto Qiagen RNeasy columns and continued using the manufacturer's instructions, incorporating the on-column DNA digest. Final RNA was eluted in a $32 \mu \mathrm{L}$ volume of RNAse-free water. We quantified total RNA using a Nanodrop and reverse transcribed 500 ng RNA using HighCapacity cDNA Reverse Transcription kit (ThermoFisher
Scientific, CA, USA). We used SYBR Green PCR Master Mix (ThermoFisher Scientific, CA, USA) to detect and quantify transcripts. Primer sequences are available upon request.

\section{Statistical analysis}

Data is represented as mean \pm standard deviation of at least 3 separate biological replicates. Utilizing Graphpad Prism, we used a Student's $t$-test when comparing 2 sets of data and Dunnet's procedure for 3 or more sets with a fixed control, with statistical significance level at $\mathrm{p}<0.05$. We determined that for an $80 \%$ powered analysis with an effect size of 5 , we required minimum 4 diabetic wounds per type of treatment for the time to closure studies.

\section{Results}

Single AFMSC dose alters diabetic wound healing time

To determine efficacy of AFMSCs in promoting wound closure, we used a validated humanized excisional wound model in adult Lepr ${ }^{\mathrm{db} / \mathrm{db}}$ type 2 diabetic mice $[9,28,30]$. Excisional wounds in Lepr ${ }^{\mathrm{db} / \mathrm{db}}$ mice demonstrate severe wound healing delays compared to their syngeneic counterparts $[9,30]$, but also respond to therapeutic interventions, making them a broadly accepted pre-clinical model $[28,31]$. In order to stimulate the events resembling human cutaneous wound healing, a silicone stent was sutured to the entire thickness of the skin, including the panniculus carnosus muscular tissue. This tissue is only present in mouse skin, unlike humans, and stenting allows for formation of granulation tissue without premature contraction of the panniculus carnosus.

We administered a single dose of $5 \times 10^{5}$ cells to each wound immediately following excision and stenting. We monitored the wound surface area, observing for loss of eschar and appearance of re-epithelialized skin. Photometric recording demonstrated that AFMSCs significantly reduce time to wound closure to $19 \pm 0.82$ days compared to vehicle (cell culture media) treated diabetic wounds at $27.5 \pm 1.30$ days $(\mathrm{p}<0.0001)$ or untreated diabetic wounds at $30.17 \pm 0.54$ days, $\mathrm{p}<0.0001$ (Fig. 1a, b). AFMSC treatment results in a $75 \%$ decrease in time to closure compared to vehicle-treated and an $80.3 \%$ decrease compared to untreated diabetic wounds. The wound closure times indicate that the AFMSCs, rather than their culture media, produce the acceleration in diabetic wound healing. We did not observe a significant difference between vehicle-treated and untreated diabetic wounds. Importantly, AFMSC treatment pushes the wound closure trajectory of diabetic wounds towards that of WT wounds (Fig. 1c). Analysis of integrated area under the curve in Fig. 1c, of remaining wound areas plotted against time, reveals the wound burden which is an indicator of wound healing capacity [32, 33]. AFMSC 


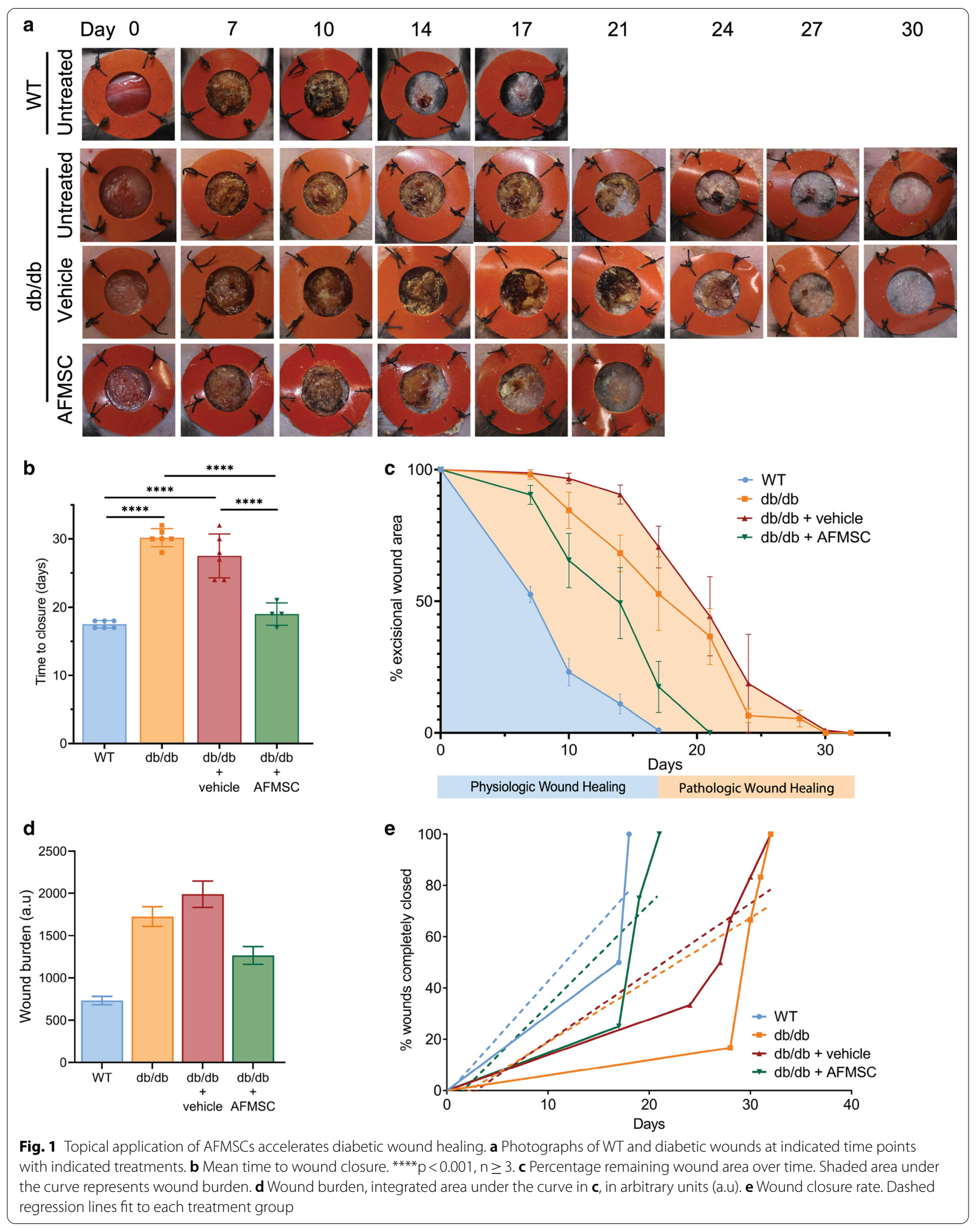


treatment reduces the wound burden of diabetic wounds, improving the chances of a favorable faster healing trajectory (Fig. 1d). We calculated a $57.7 \%$ and $46.5 \%$ decrease in wound burden of AFMSC-treated diabetic wounds, in contrast to vehicle-treated and untreated wounds, respectively. Linear regression analysis of the wound healing times per wound and type of treatment provides the rate of healing (Fig. 1e). Comparison of the slopes confirms the wound burden data, that AFMSC treatment promotes a faster healing rate than vehicle-treated or untreated diabetic wounds (Fig. 1e). Our results show that early passage AFMSCs have the capacity of promoting and normalizing diabetic wound closure.

\section{AFMSCs cannot be detected long term and do not cause adverse reactions upon administration}

Based on the success of AFMSCs in driving wound closure, we wanted to determine the length of time that AFMSCs can be detected in the diabetic wound following topical AFMSC administration. We labeled AFMSCs while still in culture with DiI, immediately prior to wound application. DiI is a lipophilic dye and we confirmed by fluorescence microscopy that AFMSCs cell membranes were labeled with the dye in our chosen conditions (Fig. 2a). Following topical administration to the diabetic wounds, we monitored the DiI fluorescence detection at regular intervals until loss of signal. Only AFMSC and not the cell culture media produced fluorescence. We detected DiI epi-fluorescence from day 0 immediately following application until $72 \mathrm{~h}$ later (Fig. 2b). The eschar already forming by this time point contributed to some autofluorescence (still visible at $96 \mathrm{~h}$ post-administration). We did not detect any DiI fluorescence in 20um diabetic wound tissue sections at post-op days 1,2 or 3 (data not shown).

We also wanted to determine whether AFMSCs trigger an immunogenic response in diabetic wounds, as the source is xenogenic. To this end, we analyzed H\&E stains of diabetic wound tissue sections from post-op days 2 and 3. AFMSCs did not cause any additional cellular influx at the time points we analyzed, compared to vehicle-treatment (Fig. 2c). Our results thus far suggest that AFMSCs do not engraft or orchestrate additional inflammatory influx in diabetic wounds.

\section{Administration of AFMSCs alters wound histology}

To further analyze the impact of AFMSC administration on cutaneous tissue repair and regeneration, we analyzed the extent of re-epithelialization based on the epithelial gap, the distance spanning the wound epidermal leading edges. Diabetic wound tissue sections collected at post-op day 7 showed that AFMSC-treated wounds had significantly reduced epithelial gap with a mean of $6.88 \pm 0.05 \mathrm{~mm}$ compared to that of vehicle-treated wounds with a mean of $8.9 \pm 0.24 \mathrm{~mm}, \mathrm{p}<0.05$ (Fig. 3a, b).

We also investigated changes in gene transcripts in diabetic wounds following treatments. Gene expression of NGF, a wound repair associated growth factor, has higher baseline expression in intact diabetic skin (Fig. 3c). In fact, the expression levels are similar to that of C57 wounded skin. Upon wounding, NGF expression significantly increases in diabetic wounds, but the AFMSC treatment normalizes the gene expressions towards that of WT wounds. We did not find any significant changes in expression of TGF $\beta$, HGF, IGF1, SDF1 or VEGF transcripts in whole wound bed tissues, though expression trends are apparent. All these factors increase in expression after wounding in WT mice, but the expression in unwounded diabetic skin is already elevated, suggesting stalled wound healing in the latter. Interestingly, AFMSCs do not express the transcripts for any of the genes analyzed, indicating that any modulations observed in the wound tissue are not due to transcriptional programs within AFMSCs. Our results indicate that the gene expression changes are induced in the wound bed cells, potentially through trophic mechanisms or cellular induction, by administration of AFMSCs.

We detected significant changes in expression of the cytokine interleukin 6 (IL6) (Fig. 3d). The unwounded or intact Lepr ${ }^{\mathrm{db} / \mathrm{db}}$ skin demonstrates significantly higher IL6 expression compared to WT intact skin, but on par with wounded WT tissues. WT wounds show a significant upregulation of IL6 in post-op day 7 wounds $(\mathrm{p}=0.0056)$ in contrast to WT intact skin. However, IL6 expression is upregulated greater than 4 -fold in vehicle-treated diabetic wounds, compared to WT wounds $(p=0.0195)$. AFMSC treatment reduces the IL6 expression in diabetic wounds such that it is no longer significantly different compared to that of a WT wound.

As IL-6 is associated with change of macrophage phenotypes [34] we analyzed macrophage populations in the wound tissue. As early as post-op day 7, arginase 1 (Arg1) expression, which indicates polarization of macrophages towards a pro-repair phenotype [35], colocalized with F4/80 + macrophages in both AFMSC-treated and vehicle-treated wound tissue sections. However, the larger granulation tissue area in AFMSC-treated diabetic wounds demonstrated significantly higher numbers of $\operatorname{Arg} 1+/ F 4 / 80+$ cells, in contrast to vehicle-treated wounds (Fig. 3e). Comparison of immunostaining on wound peripheral skin tissue sections demonstrated that similar numbers of $\mathrm{F} 4 / 80+$ macrophages are present regardless of vehicle or AFMSC treatment (Fig. 3f). Our results suggest a change in local macrophage polarization signals in response to AFMSC administration. 

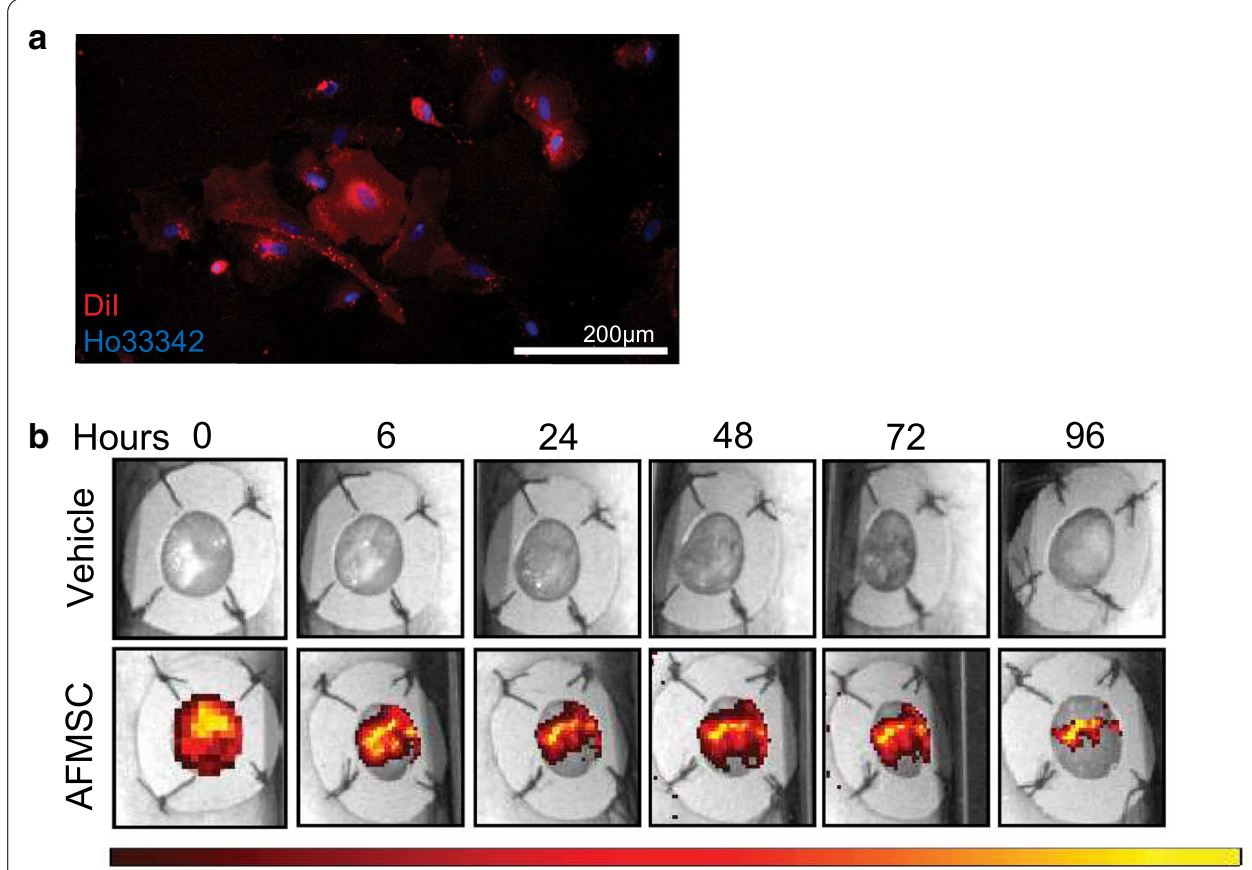

Increasing fluorescence

C
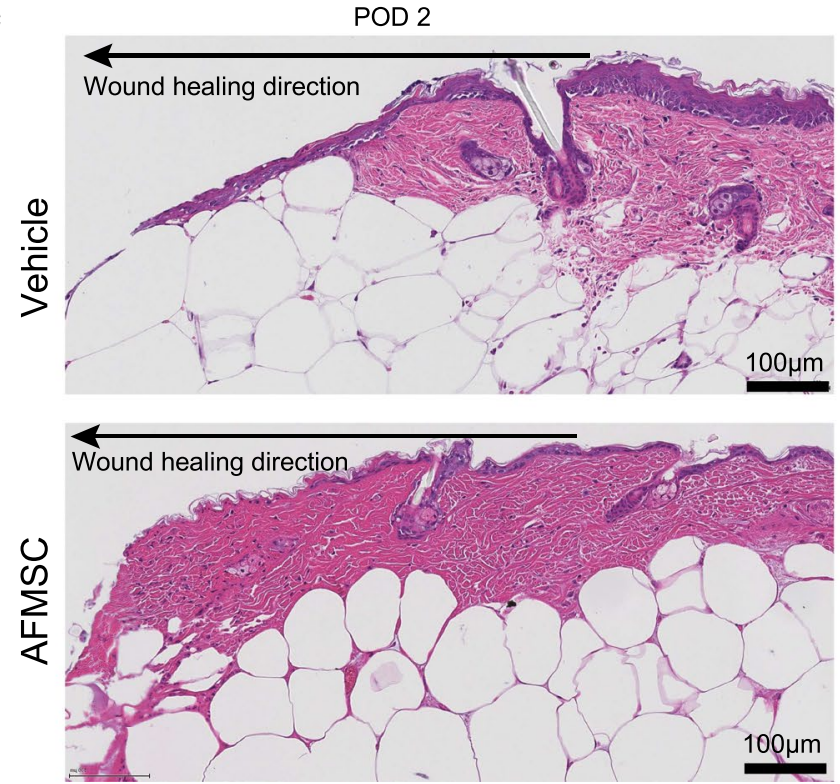
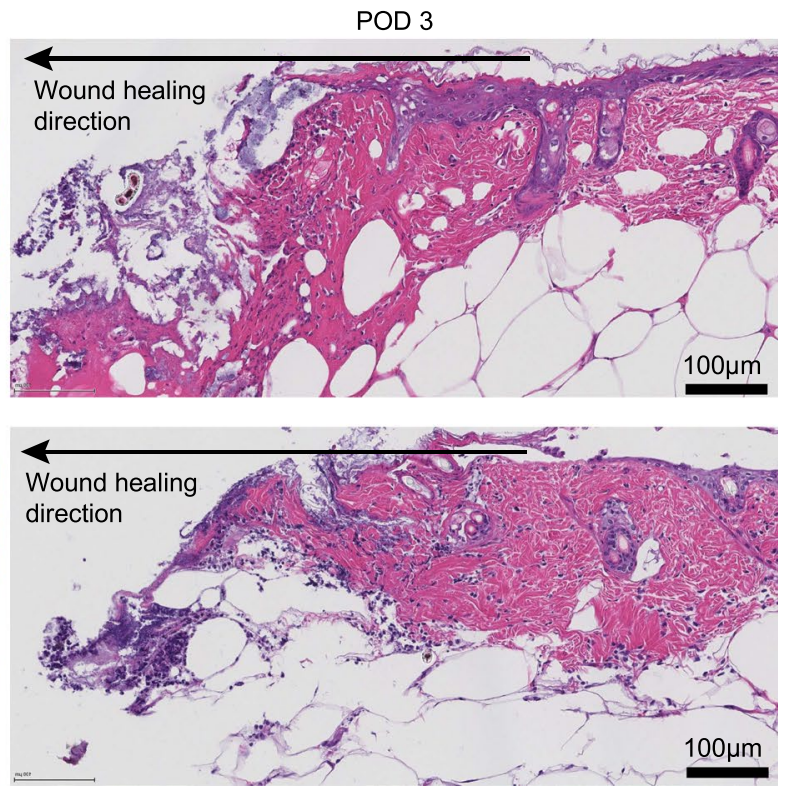

Fig. 2 AFMSCs do not induce adverse effects in mouse diabetic wounds. a Dil-stained AFMSCs in culture. b IVIS-generated images of diabetic wounds following topical application of Dil stained AFMSCs, until signal could no longer be detected. Images are representative of $n \geq 3$. $\mathbf{c}$ Representative H\&E image of diabetic wound tissue at post-operative days 2 , and 3 after vehicle treatment and AFMSC treatment, $n \geq 3$. Scale bar $100 \mu \mathrm{m}$

\section{Discussion}

A search for "mesenchymal stem cells" on clinicaltrials. gov reports more than 1100 registered human clinical trials using these cells for therapeutic purposes [36]. Evidence points towards multipotent stromal cells or MSCs having extensive remedial potential and being especially promising for tissue regeneration. MSCs, by definition, express cluster of differentiation (CD) markers including CD73, CD90, and CD105, which relates their potential to differentiate into tissues of multiple lineages [17]. 
a

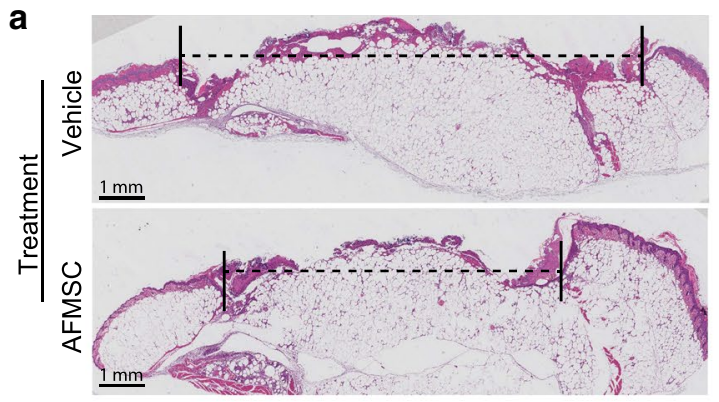

C

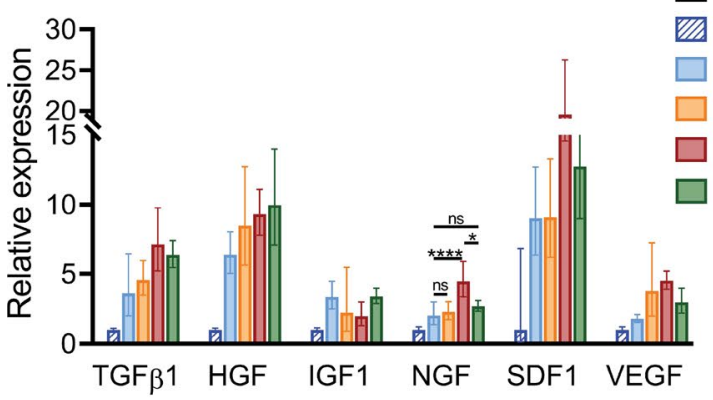

b

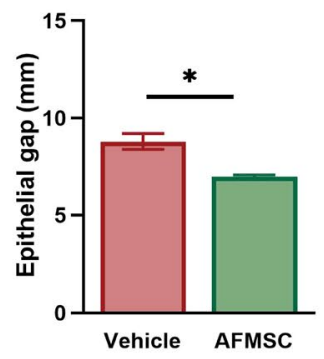

d

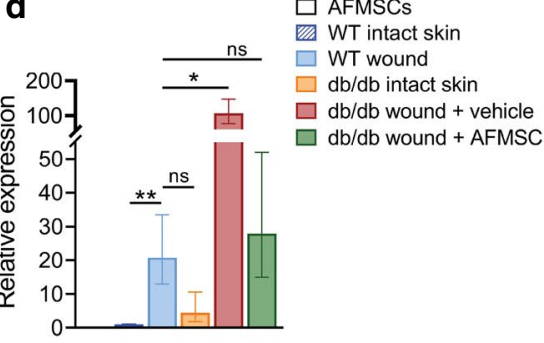

e $\quad \mathrm{db} / \mathrm{db}+$ vehicle POD 7

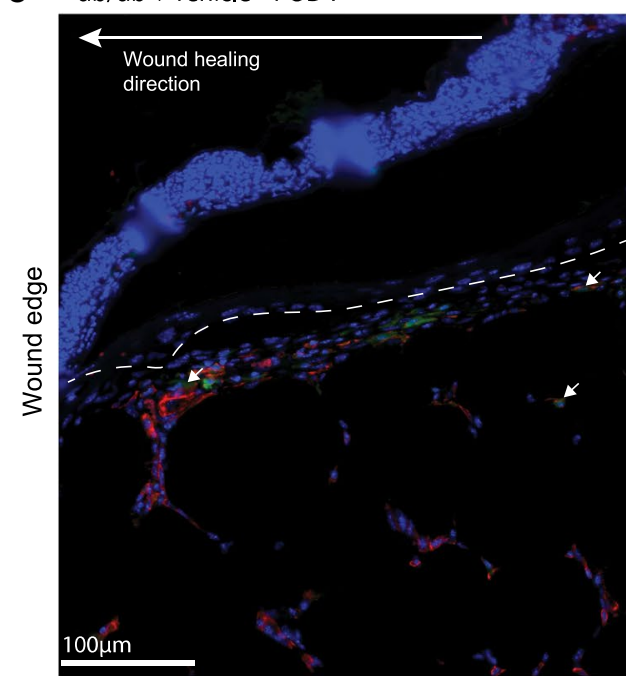

$f$
$\frac{1}{0}$
$\frac{1}{0}$
$\frac{2}{0}$
$\frac{0}{0}$
$\frac{0}{0}$
0
3

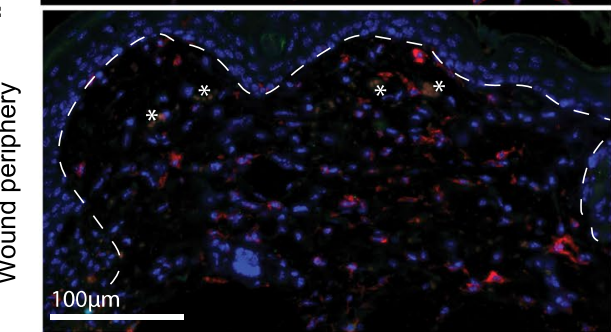

$\mathrm{db} / \mathrm{db}+$ AFMSC POD 7

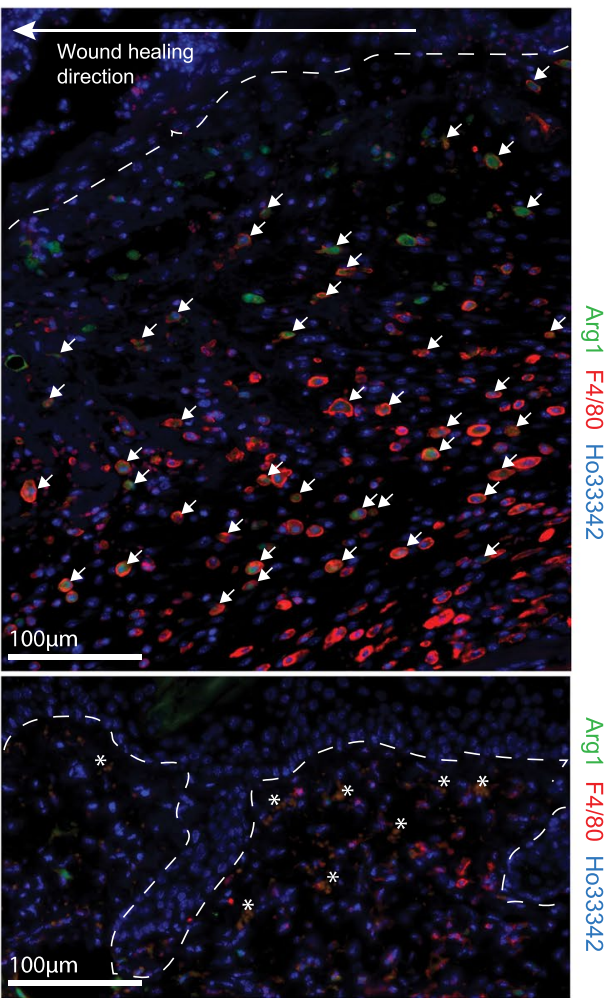

Fig. 3 AFMSC treatment induces molecular change in diabetic wound bed. a Representative H\&E tissue sections of diabetic mouse wounds from post-op day 7. Black dashed line indicates the epithelial gap. ${ }^{*} p<0.05$. b Quantification of epithelial gaps, $n \geq 3$. c Relative gene expression of wound healing factors, with mouse models and treatments as indicated at post-op day $7 .{ }^{*} p<0.05 .{ }^{* * *} p<0.0001$. ns, not significant, $n \geq 3$. $\mathbf{d}$ Relative gene expression of IL6. ${ }^{*} p<0.05$. ${ }^{* *} p<0.01$, ns, not significant, $n \geq 3$. e Representative images of immunodetection of F4/80 and Arg 1 expressing cells in diabetic wound tissues with treatments as indicated. Tissues are counterstained with Ho33342. White arrows, F4/80 $/ \mathrm{Arg} 1^{+}$cells. Asterisk, autofluorescence from erythrocytes 
Adding to this, these cells have also been found to have immunomodulatory properties [17]. These findings have led to their recognition as possible agents for mediating and enhancing the healing process, especially in environments such as diabetic ulcerations, which have abnormal immune regulation [37]. Extensive work has been done to establish both the safety and functionality of these MSCs for tissue healing. Safety studies have found that they do not result in any serious adverse effects, particularly because they have low immunogenicity [38]. With regard to functionality, studies using systemic exogenous MSCs have shown them to selectively migrate to sites of injuries, where they interact with signaling molecules [39] and also secrete factors involved in orchestrating repair [40]. With that being said, MSCs used in clinical trials are mainly derived from bone marrow (BM) and adipose tissue. However, the non-invasively sourced AFMSCs may be a better candidate for clinical applications. They also compare favorably to BM-derived MSCs because of their lower expression of MHC class I [40] and to adiposederived ones because of their greater secretion of angiogenic factors [26, 40]. Even more, amniotic fluid is easily obtained during caesarean section deliveries, and according to the latest CDC report, in 2018 alone there were over 1 million caesarean births [41]. MSCs derived from amniotic fluid have easy expansion protocols and are capable of more than 300 population doublings [13, 14, 42]. Yet, there are currently only around six clinical trials utilizing AFMSCs, none of which are for diabetic wound repair [36].

The treatment of diabetic ulcers is a unique challenge given that these wounds have multifactorial etiology. The healing process is impeded by stunted cell differentiation and proliferation, deficient extracellular matrix formation, reduced growth factor release, diminished neovascularization, and persistent expression of proinflammatory cytokines [43]. Effective therapies should facilitate complete, non-delayed wound closure and prevent reoccurrence. In the present study, we demonstrate that AFMSC therapy can ameliorate diabetic wounds by significantly decreasing the total time to wound closure. Untreated Lepr ${ }^{\mathrm{db} / \mathrm{db}}$ murine diabetic wounds take longer to heal, however, once the AFMSCs are applied, the healing time closely resembles that of WT wounds, suggesting that the typical wound healing sequence is restored (Fig. 1). As the Lepr ${ }^{\mathrm{db} / \mathrm{db}}$ mice used for this study have compromised signaling at wound sites [27], we can deduce that the allogeneic AFMSCs modulate wound healing based on their paracrine competence. Our results showing efficacy of AFMSCs corroborates previous findings in studies using rat [25] and diabetic NOD/SCID mice models [40]. We used a non-invasive AFMSC delivery strategy and applied cells to the wound microenvironment of the Lepr ${ }^{\mathrm{db} / \mathrm{db}}$ diabetic mouse skin, emulating application of the therapy immediately after debridement to expose surrounding healthy tissue. This allows for maximum contact with the wound bed cells, while eluding invasiveness. These findings showing the success of AFMSCs in treating humanized wounds in diabetic mice leads us to believe that this therapy is feasible for translational medicine.

For further clarity of therapeutic events, we analyzed the diabetic wound microenvironment post-treatment. Intriguingly, histological and molecular changes in AFMSC-treated wounds most closely related wound healing events in wild type mouse wounds. We investigated the presence and differentiation of macrophages, which are multifunctional and dynamic in the wound milieu. In the early phase, pro-inflammatory macrophages are activated due to the innate immune response [44]. In the later phases of wound healing, macrophages transition to an anti-inflammatory or pro-healing population which are involved in reestablishing integrity of the skin [35]. Their role includes support of revascularization, formation and restructuring of granulation tissue, collagen deposition and maturation, and re-epithelialization [35, 45]. Notably, macrophage plasticity is lost in chronically inflamed diabetic tissues, which have a higher presence of proinflammatory macrophages [35]. We found noteworthy modulation of IL6 expression in the diabetic wound bed as an outcome of AFMSC treatment (Fig. 3). Unwounded diabetic skin demonstrated similar IL6 transcripts as that in wounded WT skin, and AFMSC therapy successfully reduced IL6 expression of diabetic wounds to the same range as that of WT wounds. IL6 is typically recognized as a pro-inflammatory cytokine, which agrees with our findings that diabetic skin presents a hyper-inflammatory baseline, which is further upregulated upon wounding, and neutralized by AFMSC therapy. Interestingly, recent studies have suggested that IL6 could foster pro-healing macrophage polarization dependent on the microenvironment [34]. Our findings indicate that at least by 7 days after excision and therapy, AFMSCs affect macrophage differentiation to foster the transition to the later phases of healing. This offers a favorable perspective for the clinical use of AFMSCs in the treatment of diabetic ulcers.

Besides efficacy, we also examined safety of treating diabetic ulcers with AFMSCs. We did not detect any evidence of an immunogenic response in AFMSC treated mice compared to the control groups. We did not detect long term presence of AFMSCs in the mouse wound bed either. Based on our method of DiI signal detection, the loss of signal could indicate clearance of AFMSCs from the topical administration site or that the numbers of AFMSCs remaining are too low for detection by epi-fluorescence or tissue sections. The eschar that forms on the 
wounds can impede epi-fluorescent signal detection or contribute to false signals. Our findings reflect the consensus in peer-reviewed literature that MSCs and stromal cell types used for promoting repair have low to virtually no engraftment, unless in bone tissues $[5,46]$. We therefore anticipate that clinical use of this therapy should have a decreased risk of engraftment syndrome, pending clinical trial confirmation of this in long term studies.

It is important to recognize that further investigation of AFMSC secretome and paracrine activity would contribute to a better understanding of their regenerative potential. In particular, it is necessary to decipher the mechanisms by which AFMSCs normalize the macrophage populations in the diabetic wound environment. Successful clinical resolution is further dependent on whether AFMSC is effective in treating wounds of different sizes based on dosage titration. Moreover, for proficient clinical use, AFMSC manufacturing needs to be optimized. We anticipate that given the lack of immunogenicity of AFMSCs, banked cells from multiple batches will significantly increase clinical availability. We were limited in our ability to assess the safety of a scaled up AFMSC supply because we used cells from a small donor population. Nevertheless, the outcome of this study supports that AFMSCs therapy may be revolutionary for the treatment of non-healing diabetic wounds.

\section{Conclusion}

Our study demonstrates that AFMSCs show promise for promoting cutaneous wound healing in pre-clinical models of delayed wound healing. Topical and limited therapy with AFMSCs can resolve the stalled wound repair in mouse type II diabetic wounds and drive transition towards closure. AFMSCs induce molecular and cellular change in the diabetic wound microenvironment to effectively reduce time to healing of full-thickness wounds, offering a feasible translational route for the therapy of chronic wounds in patients with diabetes.

\begin{abstract}
Abbreviations
AFMSC: Amniotic fluid multipotent stromal cells; Arg1: Arginase 1; db/ $\mathrm{db}$ : Lepr ${ }^{\mathrm{db} / \mathrm{db}}$ type II diabetic mouse model; Dil: 1,1'-Dioctadecyl-3,3,3',3'tetramethylindocarbocyanine perchlorate; H\&E: Hematoxylin and eosin; HGF: Hepatocyte growth factor; Ho33342: Hoechst dye 33342; IGF1: Insulin-like growth factor 1; IL6: Interleukin 6; NGF: Nerve growth factor; POD: Post-operative day; SDF1: Stromal derived factor; TGF $\beta$ : Transforming growth factor $\beta$; VEGF: Vascular endothelial growth factor; WT: Wild type, C57 mice.
\end{abstract}

\section{Acknowledgements}

We are grateful to Dr. Bruce Young and Michael Chan for providing the AFMSCs for our study. We would also like to thank the Experimental Pathology Research Laboratory, partially supported by the Cancer Center Support Grant P30CA016087 at NYU Langone's Laura and Isaac Perlmutter Cancer Center, and the Preclinical Imaging Laboratory, also partially supported by the Laura and Isaac Perlmutter Cancer Center Support Grant NIH/NCI 5P30CA016087 and NIBIB Biomedical Technology Resource Center Grant NIH P41 EB017183.

\section{Author's contributions}

BSS and PSR composed and reviewed the manuscript, JK and PSR performed experiments, BSS, JK and PSR analyzed data, JFK, AM and SS assisted in experiments, PSR designed the study. All authors read and approved the final manuscript.

\section{Funding}

This study was partially funded by Wound Healing Foundation 3M Fellowship Award to PSR and Plastic Surgery Foundation Translational Research Grant to PSR.

\section{Availability of data and materials}

All raw data used and/or analyzed in this study are available from the corresponding author on request.

\section{Ethics approval and consent to participate}

Not applicable.

\section{Consent for publication}

Not applicable.

\section{Competing interests}

The authors declare no competing interests.

Received: 12 June 2020 Accepted: 11 December 2020

Published online: 06 January 2021

\section{References}

1. National Diabetes Statistics Report, 2020. Centers for Disease Control and Prevention, US Department of Health and Human Services, Atlanta, GA. https://www.cdc.gov/diabetes/data/statistics/statistics-report.html. Accessed 19 May 2020.

2. Hicks CW, Selvarajah S, Mathioudakis N, Sherman RE, Hines KF, Black JH 3rd, et al. Burden of infected diabetic foot ulcers on hospital admissions and costs. Ann Vasc Surg. 2016;33:149-58.

3. Sanina C, Hare JM. Mesenchymal stem cells as a biological drug for heart disease: where are we with cardiac cell-based therapy? Circ Res. 2015;117(3):229-33.

4. de Miguel MP, Prieto I, Moratilla A, Arias J, Aller MA. Mesenchymal stem cells for liver regeneration in liver failure: from experimental models to clinical trials. Stem Cells Int. 2019;2019:3945672.

5. George SK, Abolbashari M, Kim TH, Zhang C, Allickson J, Jackson JD, et al. Effect of human amniotic fluid stem cells on kidney function in a model of chronic kidney disease. Tissue Eng Part A. 2019;25(21-22):1493-503.

6. Han K-H, Kim A-K, Kim D-I. Therapeutic potential of human mesenchymal stem cells for treating ischemic limb diseases. Int J Stem Cells. 2016;9(2):163-8.

7. Rowart P, Erpicum P, Detry O, Weekers L, Grégoire C, Lechanteur C, et al. Mesenchymal stromal cell therapy in ischemia/reperfusion injury. J Immunol Res. 2015;2015:602597.

8. Hu MS, Borrelli MR, Lorenz HP, Longaker MT, Wan DC. Mesenchymal stromal cells and cutaneous wound healing: a comprehensive review of the background, role, and therapeutic potential. Stem Cells Int. 2018;2018:6901983

9. Rabbani PS, Soares MA, Hameedi SG, Kadle RL, Mubasher A, Kowzun M, et al. Dysregulation of Nrf2/Keap1 redox pathway in diabetes affects multipotency of stromal cells. Diabetes. 2019;68(1):141-55.

10. Roubelakis MG, Trohatou O, Anagnou NP. Amniotic fluid and amniotic membrane stem cells: marker discovery. Stem Cells Int. 2012;2012:107836.

11. Baghaban Eslaminejad M, Jahangir S. Amniotic fluid stem cells and their application in cell-based tissue regeneration. Int J Fertil Steril. 2012;6(3):147-56.

12. Moraghebi R, Kirkeby A, Chaves P, Rönn RE, Sitnicka E, Parmar M, et al. Term amniotic fluid: an unexploited reserve of mesenchymal stromal cells for reprogramming and potential cell therapy applications. Stem Cell Res Ther. 2017;8(1):190.

13. Young BK, Chan MK, Liu L, Basch RS. Amniotic fluid as a source of multipotent cells for clinical use. J Perinat Med. 2016;44(3):333-7. 
14. Larson A, Gallicchio V. Amniotic derived stem cells: role and function in regenerative medicine. J Cell Sci Ther. 2017;8:3.

15. Dziadosz M, Basch RS, Young BK. Human amniotic fluid: a source of stem cells for possible therapeutic use. Am J Obstet Gynecol. 2016;214(3):321-7.

16. Sun Q, Li F, Li H, Chen R-H, Gu Y-Z, Chen Y, et al. Amniotic fluid stem cells provide considerable advantages in epidermal regeneration: $\mathrm{B} 7 \mathrm{H} 4$ creates a moderate inflammation microenvironment to promote wound repair. Sci Rep. 2015;5:11560.

17. Brown C, McKee C, Bakshi S, Walker K, Hakman E, Halassy S, et al. Mesenchymal stem cells: cell therapy and regeneration potential. J Tissue Eng Regen Med. 2019;13(9):1738-55.

18. Perin L, Sedrakyan S, Da Sacco S, De Filippo R. Characterization of human amniotic fluid stem cells and their pluripotential capability. Methods in cell biology, vol. 86. USA: Academic Press; 2008. p. 85-99.

19. You Q, Tong X, Guan Y, Zhang D, Huang M, Zhang Y, et al. The biological characteristics of human third trimester amniotic fluid stem cells. J Int Med Res. 2009;37(1):105-12.

20. Loukogeorgakis SP, De Coppi P. Concise review: amniotic fluid stem cells: the known, the unknown, and potential regenerative medicine applications. Stem Cells. 2017;35(7):1663-73.

21. Moschidou D, Mukherjee S, Blundell MP, Drews K, Jones GN, Abdulrazzak $\mathrm{H}$, et al. Valproic acid confers functional pluripotency to human amniotic fluid stem cells in a transgene-free approach. Mol Ther. 2012;20(10):1953-67.

22. Vlahova F, Hawkins KE, Ranzoni AM, Hau K-L, Sagar R, De Coppi P, et al. Human mid-trimester amniotic fluid (stem) cells lack expression of the pluripotency marker OCT4A. Sci Rep. 2019;9(1):8126.

23. Klemmt PAB, Vafaizadeh V, Groner B. The potential of amniotic fluid stem cells for cellular therapy and tissue engineering. Exp Opin Biol Ther. 2011;11(10):1297-314.

24. Dolin CD, Chan MK, Basch RS, Young BK. Human term amniotic fluid: a novel source of stem cells for regenerative medicine. Am J Obstet Gynecol. 2018;219(3):308-9.

25. Yang JD, Choi DS, Cho YK, Kim TK, Lee JW, Choi KY, et al. Effect of amniotic fluid stem cells and amniotic fluid cells on the wound healing process in a white rat model. Arch Plast Surg. 2013;40(5):496-504

26. Yoon BS, Moon J-H, Jun EK, Kim J, Maeng I, Kim JS, et al. Secretory profiles and wound healing effects of human amniotic fluid-derived mesenchymal stem cells. Stem Cells Dev. 2009;19(6):887-902.

27. Brem H, Tomic-Canic M. Cellular and molecular basis of wound healing in diabetes. J Clin Invest. 2007;117(5):1219-22.

28. Galiano RD, Michaels JT, Dobryansky M, Levine JP, Gurtner GC. Quantitative and reproducible murine model of excisional wound healing. Wound Repair Regen Off Publ Wound Heal Soc Eur Tissue Repair Soc. 2004;12(4):485-92.

29. Margolis DJ, Bilker W, Boston R, Localio R, Berlin JA. Statistical characteristics of area under the receiver operating characteristic curve for a simple prognostic model using traditional and bootstrapped approaches. J Clin Epidemiol. 2002;55(5):518-24.

30. Rabbani PS, Zhou A, Borab ZM, Frezzo JA, Srivastava N, More HT, et al. Novel lipoproteoplex delivers Keap1 siRNA based gene therapy to accelerate diabetic wound healing. Biomaterials. 2017:132:1-15.
31. Michaels J, Churgin SS, Blechman KM, Greives MR, Aarabi S, Galiano RD, et $\mathrm{al}$. $\mathrm{db} / \mathrm{db}$ mice exhibit severe wound-healing impairments compared with other murine diabetic strains in a silicone-splinted excisional wound model. Wound Repair Regen. 2007;15(5):665-70

32. Margolis DJ, Allen-Taylor L, Hoffstad O, Berlin JA. Diabetic neuropathic foot ulcers: predicting which ones will not heal. Am J Med. 2003;115(8):627-31.

33. Margolis DJ, Allen-Taylor L, Hoffstad O, Berlin JA. The accuracy of venous leg ulcer prognostic methods in a wound care system. Wound Repair Regen. 2004;12(2):163-8.

34. Fernando MR, Reyes JL, lannuzzi J, Leung G, McKay DM. The pro-inflammatory cytokine, interleukin-6, enhances the polarization of alternatively activated macrophages. PLoS ONE. 2014;9(4):e94188.

35. Krzyszczyk P, Schloss R, Palmer A, Berthiaume F. The role of macrophages in acute and chronic wound healing and interventions to promote prowound healing phenotypes. Front Physiol. 2018;9:419.

36. United States National Library of Medicine. Clinicaltrials.gov. http://clini caltrials.gov. Accessed 19 May 2020

37. Larouche J, Sheoran S, Maruyama K, Martino MM. Immune regulation of skin wound healing: mechanisms and novel therapeutic targets. Adv Wound Care (New Rochelle). 2018;7(7):209-31.

38. Lalu MM, McIntyre L, Pugliese C, Fergusson D, Winston BW, Marshall JC, et al. Safety of cell therapy with mesenchymal stromal cells (SafeCell): a systematic review and meta-analysis of clinical trials. PLoS ONE. 2012;7(10):e47559.

39. Wei X, Yang X, Han Z-p, Qu F-f, Shao L, Shi Y-f. Mesenchymal stem cells: a new trend for cell therapy. Acta Pharmacol Sin. 2013;34(6):747-54.

40. Kim S-W, Zhang H-Z, Guo L, Kim J-M, Kim MH. Amniotic mesenchymal stem cells enhance wound healing in diabetic NOD/SCID mice through high angiogenic and engraftment capabilities. PLOS ONE. 2012;7(7):e41105.

41. National Center for Health Statistics: Births-Method of Delivery. Centers for Disease Control and Prevention, US Department of Health and Human Services, Atlanta, GA. https://www.cdc.gov/nchs/fastats/delivery. htm. Accessed 19 May 2020.

42. Joo S, Ko IK, Atala A, Yoo JJ, Lee SJ. Amniotic fluid-derived stem cells in regenerative medicine research. Arch Pharmacal Res. 2012;35(2):271-80

43. Yang M, Sheng L, Zhang TR, Li Q. Stem cell therapy for lower extremity diabetic ulcers: where do we stand? Biomed Res Int. 2013;2013:462179.

44. Martinez FO, Gordon S. The M1 and M2 paradigm of macrophage activation: time for reassessment. F1000Prime Rep. 2014;6:13.

45. Ganesh GV, Ramkumar KM. Macrophage mediation in normal and diabetic wound healing responses. Inflamm Res. 2020;69(4):347-63.

46. De Coppi P, Bartsch G, Siddiqui MM, Xu T, Santos CC, Perin L, et al. Isolation of amniotic stem cell lines with potential for therapy. Nat Biotechnol. 2007:25(1):100-6.

\section{Publisher's Note}

Springer Nature remains neutral with regard to jurisdictional claims in published maps and institutional affiliations.

Ready to submit your research? Choose BMC and benefit from

- fast, convenient online submission

- thorough peer review by experienced researchers in your field

- rapid publication on acceptance

- support for research data, including large and complex data types

- gold Open Access which fosters wider collaboration and increased citations

- maximum visibility for your research: over 100M website views per year

At BMC, research is always in progress.

Learn more biomedcentral.com/submissions 